\title{
Experimental model of Low Power NAS and Cloud drive based on Raspberry Pi
}

\author{
I. Kandov
}

\begin{abstract}
Mobile technologies and in particular the use of cloud space are successfully integrated on the web and their users are increasing every day. The idea of cloud is that each user has his own place to store information that can be access from anywhere. Much of the cost of the service depends on the amount of space used. The idea is to use the Raspberry PI as a network storage, that later can be used as cloud server.
\end{abstract}

Index Terms - Raspberry, cloud, Network storage system,

\section{INTRODUCTION}

$\mathrm{B}$ ASED on Raspberry Pi and his idea of the creation, we can realize various complex devices at significantly lower cost. Used architecture, good CPU performance, and ease of adding additional devices makes it easy to use. 32 bit processor, $\mathrm{RPi}$ easily able to deal with applications that deal and PCs. The level of integration of the modules is very high. There is a high number of active modules for managing peripherals.

The description of all the important modules is presented in Figure 1.

To create a network storage except Raspberry $\mathrm{Pi}$ is necessary and enough hard drive in which information is stored Ill. By using a USB hub, we can add an unlimited number of storage devices

\section{II.EXPERIMENTAL MODEL}

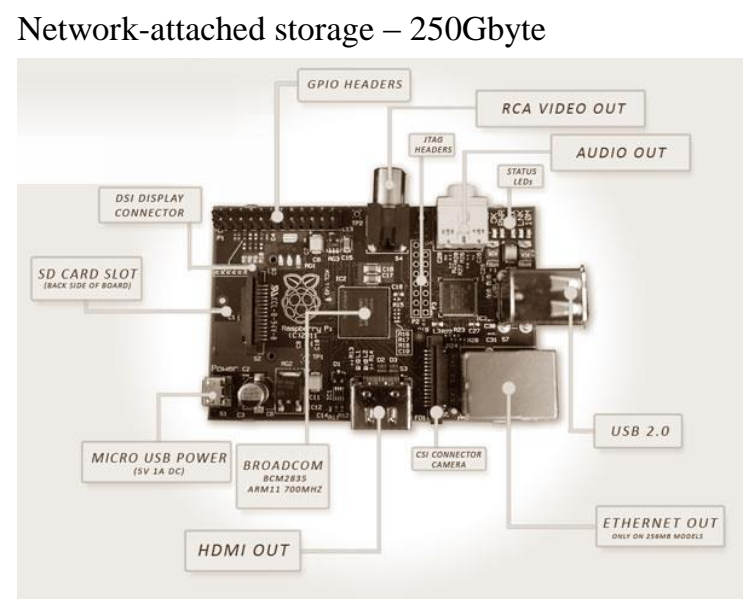

Fig. 1. Description of Raspberry PI
For the realization of networked storage are needed:

- Raspberry Pi (RPi)

- SD card class 10

- External memorable device - (HDD)

- Wireless or wired connection to the LAN.

The idea itself is network storage for storing and sharing information with other computers on the local network, and the information that can be stored is no restriction on the format. Files to be stored on RPi, can be easily accessed by local network. Devices that can work with them no restriction on operating system - Windows, Mac Os, Linux and smart phone and tablets.

To execute the project is necessary Raspberry $\mathrm{Pi}$ be configured as a Samba server, and thus provide the files via Samba client to users on the network. Samba provides file and print services for various Microsoft Windows clients and can integrate with a Windows Server domain, either as a Primary Domain Controller (PDC) or as a domain member. It can also be part of an Active Directory domain.

Samba protocol was used only in windows as it was called Server message Block, but is now known under other operating systems. The installation of Samba is very Lens and is preferred over FTP, but if a fault is determined, it is not recommended to implement high load.

You need to be an admin user to save any changes when you're finished editing [1].

In our case any command which may change the server configuration needs to be carried out by the admin user. Since for security reasons you cannot login as root (the admin user) the alternative is to insert sudo before every command then provide the admin password, this keeps things nice and secure.

Select Samba and SSH during the installation procedure. The installed server has no graphical interactive interface. During installation you installed SSH and having SSH running on in default mode can be a risk. Have to change the default port to a random one.

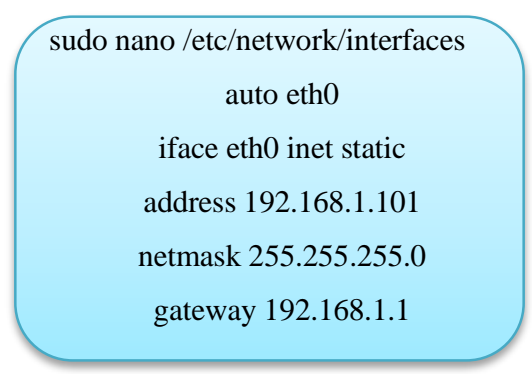

Iskren Kandov is with the Faculty of Electrical Engineering, Technical University of Gabrovo, Bulgaria (e-mail:iskren.kandov@gmail.com). 
The default port is 22 which many people don't bother changing so this is the one used by hackers and hacking software to try and gain access to your server [1,2,6].

If the IP address is not static during installation it will have a DHCP address assigned to it by your router. This would be fine if the router is never been rebooted, or the routed is shutdown. High load means watching videos, implementation of a large number of requests and more. The default configuration file allows users to view their home directories as a Samba share $[5,7]$.

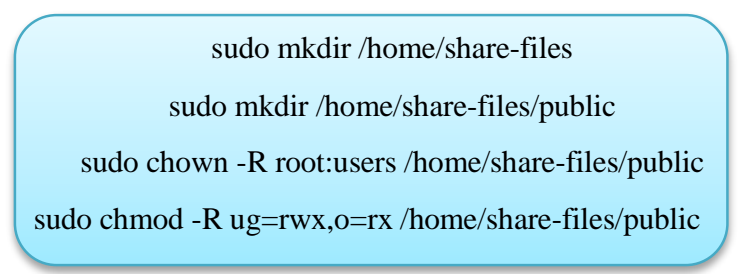

\section{ADDING AN EXTERNAL HDD}

Adding an external hard drive is done via the USB port of Raspberry, as to be able to recognize the need to initialize [3].

\section{$/ \mathrm{dev} / \mathrm{sda} 1$}

Under Linux finding additional HDD is done with this code, the last two characters show how we have incorporated additional devices.

sudo mkdir /home/share-files/public/drive

sudo mount /dev/sdxx /home/share-files/public/drive

After adding of the device in the directory is necessary Samba server is restarted, and to install the client version of all the computers participating in the network. Access to network storage is done by filling in the username and password are added to each user [4].

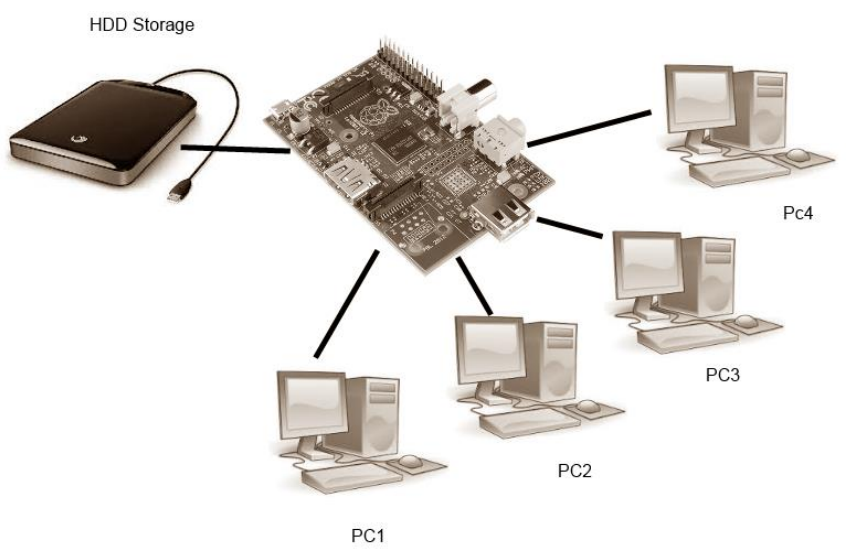

Fig.2. Raspberry PI network attached storage

\section{OWN CLOUD}

The idea of cloud space is the ability for remote access. Displayed in Figure 3 is the addition of cloud space. For the implementation of cloud space use OwnCloud application. Through it we can add files to access such that uses automatic synchronization and more.

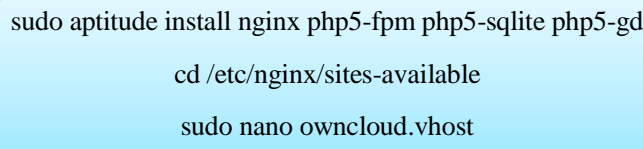

After installing php5 and add permission to a website need to download and configure the application itself.

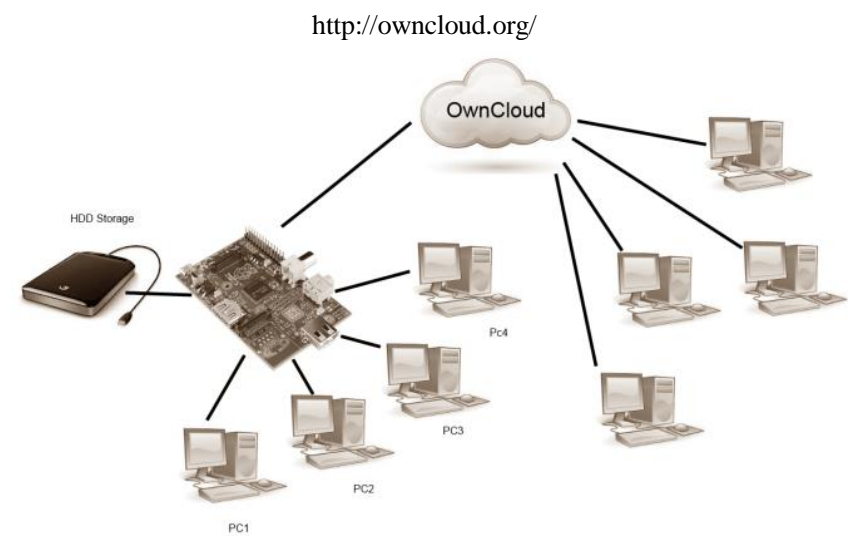

Fig.3. Own Cloud Raspberry PI

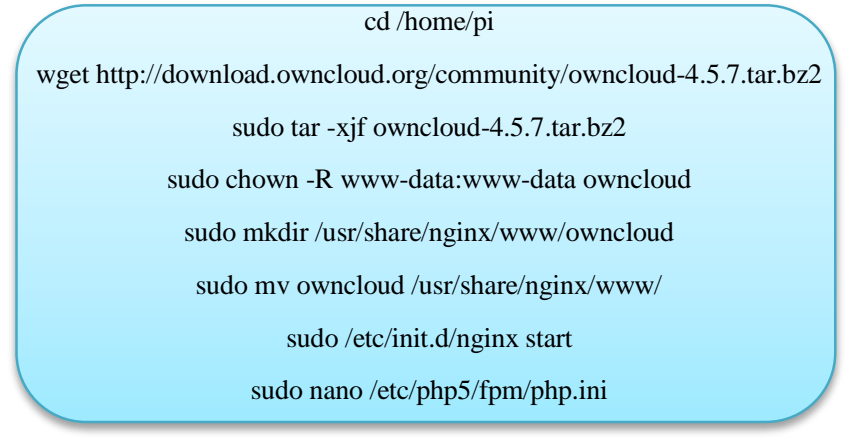

It is to be determined and the maximum file size that can be added.

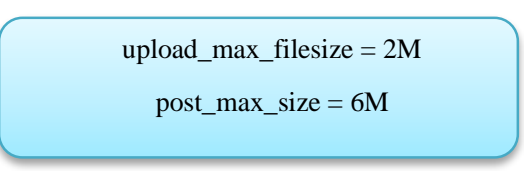

after that the system needs to reboot:

Sudo reboot now

\section{RESUlts}

On Figure 4 is shown the real live test model. Using an external HDD and additional SATA convertor with external power supply [4].

Own Cloud has an interactive graphical design. The application is useable through web browser. It consists its own

Drag and drop function commonly based on java script on similar. The look of the cloud is shown in Figure 5.

Combination between Cloud and NAS gives users opportunity for choosing how and where to upload they files. Cloud technologies means that every computer with internet is your computer. 


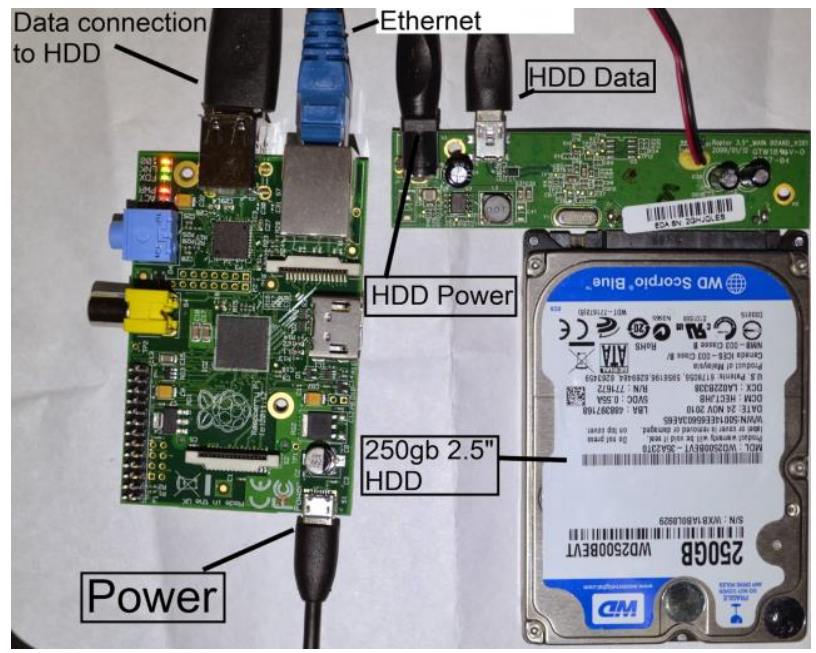

Fig.IV. Raspberry PI NAS model

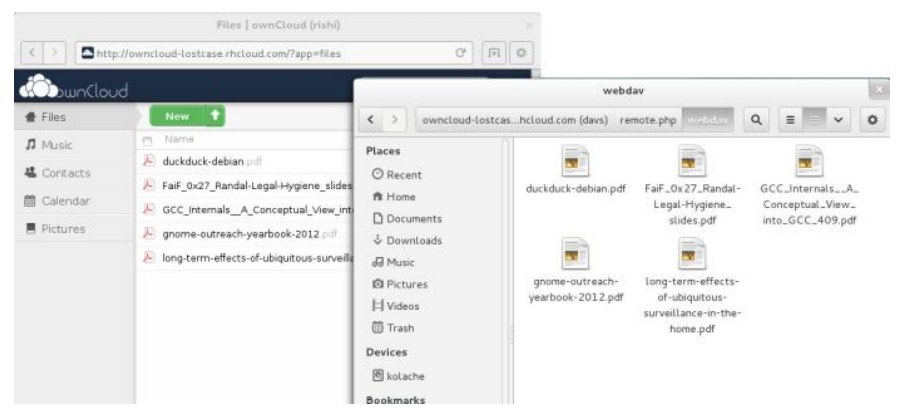

Fig.V. Installed Own Cloud

The timing chart of traffic in, traffic out downloadtime and packets moved to and from the cloud server are shown in Figure 6 [10].

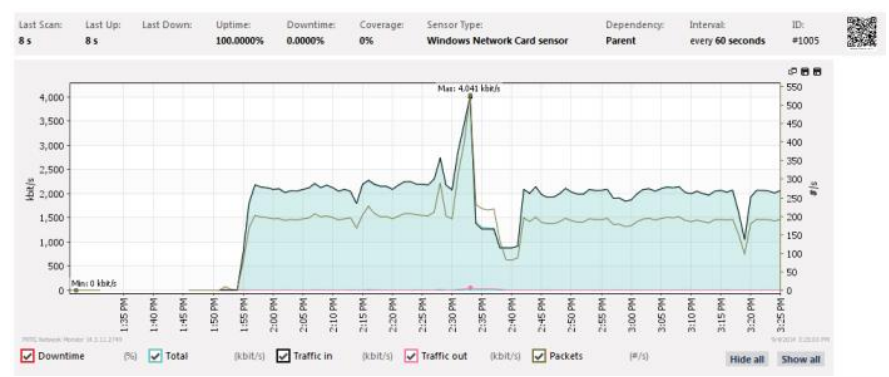

Fig.VI. Traffic Timing Chart

\section{REFERENCES}

[1] James F. Kurose, Keith W. Ross - Computer Networking, Aways Learning - 2012.

[2] James F. Kurose \& Keith W. Ross - Computer Networking: A Top-Down Approach Featuring the Internet, Boston, MA : Pearson Education, Inc., 2005.

[3] Kanev J., Sadinov S., "Analyzing RTSP protocol features for managing, video on demand server”, UNITECH 2012, Gabrovo Bulgaria, 2012.
[4] Kanev J., Exaple of Live TV Streaming Server Based on Raspberry PI", Unitech 2013, Gabrovo Bulgaria, 2013.

[5] Richard Blum - Beginning Linux Programming, John Wiley \& Sons; 4th Edition edition (Nov 2007).

[6] http://www.cisco.com/c/en/us/td/docs/iosxml/ios/ipaddr_dhcp/configuration/12-4t/dhcp-12-4t-book.pdf - 2014.

[7] https://help.ubuntu.com/10.04/serverguide/samba-fileserver.html - 2014

[8] http://owncloud.org/ - 2014.

[9] http://www.raspberrypi.org/ - 2014

[10] http://netsimk.com/ - 2014

\section{ACKNOWLEDGMENT}

The present document has been produced with the financial assistance of the European Social Fund under Operational Programme "Human Resources Development". The contents of this document are the sole responsibility of "Angel Kanchev" University of Ruse and can under no circumstances be regarded as reflecting the position of the European Union or the Ministry of Education and Science of Republic of Bulgaria. Project № BG051PO001-3.3.06-0008 "Supporting Academic Development of Scientific Personnel in Engineering and Information Science and Technologies".

\section{BIOGRAPHY}

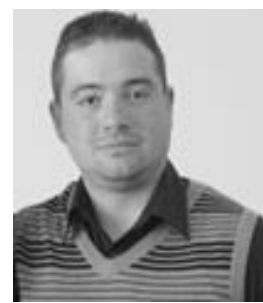

ISKREN KANDOV was born in Gabrovo, Bulgaria, in 1988. He received master degrees in electronics from the Technical University of Gabrovo, Bulgaria, in 2012.

His research interests are sensor networks, web based system, mobile technologies for control and measure electrical parameters and cloud system for storing information from sensor networks. 